Please do not remove this page

RMIT

UNIVERSITY

\title{
On the relativistic Doppler effect for precise velocity determination using GPS
}

Zhang, Jason; Zhang, Kefei; Grenfell, Ronald; Deakin, Rodney

https://researchrepository.rmit.edu.au/esploro/outputs/9921859439301341/filesAndLinks?institution=61RMIT_INST\&index=null

Zhang, J., Zhang, K., Grenfell, R., \& Deakin, R. (2006). On the relativistic Doppler effect for precise velocity determination using GPS. Journal Of Geodesy, 80, 104-110. https://doi.org/10.1007/s00190-006-0038-8

Document Version: Accepted Manuscript

Published Version: https://doi.org/10.1007/s00190-006-0038-8

Repository homepage: https://researchrepository.rmit.edu.au

(c) Springer-Verlag 2006

Downloaded On 2023/04/26 18:28:12 +1000 


\title{
On the Relativistic Doppler Effect for Precise Velocity Determination using GPS
}

\author{
JASON ZHANG, KeFEI ZHANG, Ron GRENFELL, RoD DEAKIN \\ School of Mathematical and Geospatial Sciences, RMIT University, Melbourne, \\ VIC 3000, Australia.
}

Email: kefei.zhang@rmit.edu.au

Tel: +61-3-99253272

Fax: +61-3-96632517

\begin{abstract}
The Doppler Effect is the apparent shift in frequency of an electromagnetic signal that is received by an observer moving relative to the source of the signal. The Doppler frequency shift relates directly to the relative speed between the receiver and the transmitter, and has thus been widely used in velocity determination. A GPS receiver-satellite pair is in the Earth's gravity field and GPS signals travel at the speed of light, hence both Einstein's special and general relativity theories apply.
\end{abstract}

This paper establishes the relationship between a Doppler shift and a user's ground velocity by taking both the special and general relativistic effects into consideration. A unified Doppler shift model is developed, which accommodates both the classical Doppler Effect and the relativistic Doppler Effect under special and general relativities. By identifying the relativistic correction terms in the model, a highly accurate GPS Doppler shift observation equation is presented. It is demonstrated that in the GPS "frequency" or "velocity" domain, the relativistic effect from satellite motion changes the receiver-satellite line-of-sight direction, and the measured Doppler shift has correction terms due to the relativistic effects of the receiver potential difference from the geoid, the orbit eccentricity, and the rotation of the Earth.

\section{Key words}

GPS velocity determination, Doppler shifts, Doppler Effects, relativistic Doppler Effects, gravitational potential, satellite orbit.

\section{Introduction}

For high accuracy velocity determination, GPS satellites are taken as known space moving signal sources from which a GPS receiver senses Doppler shifts due to the relative motion between the observed satellites and the receiver. The velocity of the GPS receiver can then be determined if Doppler shifts from more than four GPS satellites have been measured. Without Selective Availability, $\pm 0.2 \mathrm{~m} / \mathrm{sec}$ per axis (95\%) accuracy is guaranteed by the GPS system for standalone GPS users (DoD 1996). Ground velocity accuracy of sub-centimetre per second level, however, is now achievable in standalone mode (van Graas and Soloview 2003; Serrano et al. 2004). 
It is well known that the GPS observables have errors and biases that are characterised as having correlations spatially and temporally. Differential GPS techniques provide an efficient solution to account for the correlated errors and biases, since many un-modelled errors may be cancelled out by the differential process. In order to achieve high accuracy in a standalone mode, all errors associated with the observations are required to be well modelled and properly corrected for. To pursue velocity accuracy in millimetres per second level, it is required to carefully take account for error sources at $0.1 \mathrm{~mm} / \mathrm{s}$ level in range rate measurements.

This paper highlights issues on the precise velocity determination using GPS in a standalone mode, focusing on the inherent relativistic effects on GPS Doppler observations. It begins with an introduction of the classical Doppler Effect and velocity determination, and then extends the classical Doppler Effect to the relativistic Doppler Effect which considers the special relativity induced by the high speed of satellites in orbit. Due to the Earth's gravity field, Einstein's general relativity theory needs to be considered in GPS applications. In order to take both special and general relativity into account, the generic equation given by Ashby (2003, p.27) is modified to a practical form, from which a highly accurate theoretical GPS Doppler shift model is developed. A rigorous proof is also provided to the above-modified equation in the Appendix. By considering all major error sources in GPS measurements, a precise Doppler shift observation equation is presented. It is demonstrated in the derivation that the direction of the range rate vector is changed due to the relativistic effect from satellite motion, and the measured Doppler shift has correction terms due to the relativistic effects of the receiver potential difference from the geoid, the orbit eccentricity, and the Earth's rotation.

A comprehensive relativistic error analysis is performed with identification and verification. With the proposed Doppler shift measurement model, it is expected that a receiver's velocity can be accurately determined in either static or highly dynamic applications.

\section{Doppler Effect and Relative Velocity}

\subsection{Classical Doppler Effect}

A Doppler effect is experienced whenever there is relative motion between source and observer of wave signals. It is a phenomenon common to all harmonic waves (Serway 1996). In the context of GPS, the classic Doppler Effect is expressed as (Misra and Enge 2001, p.16)

$$
f_{r}=f_{s} \cdot\left(1-\frac{\dot{\vec{r}}_{r}^{s}}{c}\right)
$$

where superscript $s$ denotes the transmitter on a satellite; subscript $r$ represents a receiver; $f_{r}$ is the received frequency from the receiver; $f_{s}$ is the original frequency of 
the transmitter; $c$ represents the speed of light in vacuum; $\vec{r}_{r}^{s}$ is the receiver-satellite line-of-sight (LOS) vector; and $\dot{\vec{r}}_{r}^{s}$ is the relative velocity between the signal transmitter on the satellite and the receiver in the LOS direction.

The change of the frequency is termed as the Doppler shift $D$, which can be related to the relative velocity by rewriting (1) into the following form

$D \equiv\left(f_{r}-f_{s}\right)=f_{s} \frac{\dot{\vec{r}}_{r}^{s}}{c}=\frac{1}{\lambda_{s}} \dot{\vec{r}}_{r}^{s}$

where, $\lambda_{s}=\frac{c_{0}}{f}$ is the signal wavelength. Note that the Doppler shift $D$ has a positive sign when the receiver and the transmitter approach each other and has a negative sign when they depart from each other under this definition. It is this form that has been widely used in GPS velocity determination (Misra and Enge 2001; HofmannWellenhof et al. 2001).

\subsection{Relativistic Doppler Effect (Special Relativity Only)}

As a GPS satellite orbits the Earth at a high speed (approximately $3.8 \mathrm{~km} / \mathrm{s}$ ), transmitting electromagnetic wave signals that travel at the speed of light, Einstein's special relativity theory applies. The relativistic Doppler effect is well known to the GPS literature (Wells et al. 1987; Seeber 1993; Seeber 2003), wherein the ratio of received and transmitted frequencies of GPS signals is given as (cf. Seeber 1993, p.280)

$$
\frac{f_{r}}{f_{s}}=\frac{1-(v \cdot \cos \beta) / c}{\sqrt{1-\frac{v^{2}}{c^{2}}}}=\frac{1-\frac{\dot{\vec{r}}}{c}}{\sqrt{1-\frac{v^{2}}{c^{2}}}}
$$

where $v$ is the tangential velocity of a GPS satellite; $\beta$ is the angle between the satellite tangential velocity vector and the vector in the direction to the GPS receiver.

Equation (3) can be expanded using binomial series

$$
f_{r}=f_{s} \cdot\left(1-\frac{\dot{\vec{r}}}{c}\right) \cdot\left(1+\frac{v^{2}}{2 c^{2}}+\frac{v^{4}}{8 c^{4}}+\cdots\right)
$$

when the higher order terms ( $v / c$ terms equal to or greater than second order) in (4) are neglected, the Doppler shift becomes

$$
D \equiv f_{r}-f_{s}=\frac{\dot{\vec{r}}}{c} f_{s}=\frac{1}{\lambda} \cdot \dot{\vec{r}}
$$

where once again we designate $D$ as positive when a satellite is approaching and negative when the satellite is departing.

Equation (3) is the relativistic Doppler Effect equation under the special relativity theory. When the satellite velocity $v<<c$, the denominator in (3) is approximately 
unity and gives the classical Doppler Effect in the form of (1). Hence the relativistic Doppler Effect is in a more generic form.

Among the neglected terms, only the second order term may have some numerical effect on the frequency reception. Seeber (2003) termed it as the "transversal" Doppler effect, whereas Ashby and Spilker (1996) named it as the "second-order Doppler effect". The magnitude of this effect may be evaluated as follows

$\Delta f_{r} \equiv f_{s} \cdot\left(1-\frac{\dot{\vec{r}}}{c}\right) \cdot \frac{v^{2}}{2 c^{2}}=\frac{f_{s} v^{2}}{2 c^{2}}-\frac{f_{s} \dot{\vec{r}} v^{2}}{2 c^{3}} \approx \frac{f_{s}}{2} \cdot \frac{v^{2}}{c^{2}}$

Since GPS signals have very high frequencies $(\mathrm{L} 1=1575.42 \mathrm{MHz}$, $\mathrm{L} 2=1227.60 \mathrm{MHz}$ ) and $\Delta f_{r}>0.1 \mathrm{~Hz}$ which is equivalent to more than $2 \mathrm{~cm} / \mathrm{s}$ error in the range rate, the second-order Doppler Effect should be taken into consideration for precise applications.

\subsection{GPS Relativistic Doppler Effect}

The GPS signal, however, propagates in the Earth's gravity field, thus both the special and general relativities apply for the Doppler Effects. Ashby (2003, p.27) gives the received GPS signal frequency in the form of

$$
f_{r}=f_{0} \cdot\left[1+\frac{-V_{r}+\dot{\vec{r}}_{r}^{2} / 2+\Phi_{0}+2 G M / a_{o r b}+2 V^{s}}{c^{2}}\right] \cdot \frac{1-\vec{n}_{r}^{s} \cdot \dot{\vec{r}}_{r} / c}{1-\vec{n}_{r}^{s} \cdot \dot{\vec{r}}^{s} / c}
$$

where $\Phi_{0}$ is the gravity potential on the geoid, including the centrifugal potential from the Earth's rotation; the geoidal potential $\Phi_{0}$ is a theoretical constant and it has been proposed to define a unique World Height System (Kouba 2002; Kouba 2004); $\Phi_{0} / c^{2}=6.969290134 \times 10^{-10}$ was defined as the terrestrial time scale in the IAU resolutions adopted at the $24^{\text {th }}$ General Assembly (IAU Res 2000); $f_{0}$ is the GPS nominal frequency; $G M$ is the product of the Universal constant of Gravitation $G$ and the earth's mass $M . G M=(3986004.418 \pm 0.008) \times 10^{8}\left(\mathrm{~m}^{3} / \mathrm{s}^{2}\right)$ is adopted for the World Geodetic System 1984 (WGS84) (NIMA 2000, p.3-3); $a_{o r b}$ is the semi-major axis of the satellite elliptical orbit; $\vec{n}_{r}^{s}$ is the receiver to satellite LOS unit vector; $V$ is the Newtonian gravitational potential (mass attraction only) at a given position, the subscript $r$ and superscript $s$ are assigned to represent the potentials of receiver and satellite respectively; the GPS satellite's gravitational potential can be simply approximated by taking the Earth as a point mass, while the receiver gravitational potential can be approximated by taking into account the Earth equatorial oblateness as follows

$V=-\frac{G M}{\|\vec{r}\|}\left[1-J_{2}\left(\frac{a_{e}}{\|\vec{r}\|}\right)^{2} P_{2}(\sin B)\right]$

where $J_{2}=1.08262998905 \mathrm{H}_{10} 0^{-3}$ is the Earth's second zonal harmonic coefficient, also known as the dynamical form factor; $B$ is the latitude of receiver; $P_{2}$ is the Legendre polynomial of degree two, $\mathrm{P}_{2}(\mathrm{x})=0.5\left(3 \mathrm{x}^{2}-1\right)$; and $a_{e}$ is the semi-major axis of WGS84 ellipsoid; $\|\vec{r}\|$ is the distance from the point of interest to the Earth's centre. 
It should be noted that in a rotating Earth-Centred-Earth-Fixed (ECEF) coordinate system, to apply (7) requires the centrifugal potential of the receiver be introduced (Audoin and Guinot 2001). In the ECEF system, the velocity of a receiver in ground applications are relatively small when comparing with the speed of light, thus the kinematic energy term of the receiver can be safely neglected. As such, we have a modified form as

$$
\begin{aligned}
f_{r} & =f_{0} \cdot\left[1+\frac{-V_{r}+1 / 2 \cdot \omega^{2}\left\|\vec{r}_{r}\right\|^{2}(\cos B)^{2}+\Phi_{0}+2 G M / a_{o r b}+2 V_{s}}{c^{2}}\right] \cdot \frac{1-\vec{n}_{r}^{s} \cdot \dot{\vec{r}}_{r} / c}{1-\vec{n}_{r}^{s} \cdot \dot{\vec{r}}^{s} / c} \\
& =f_{0} \cdot\left[1+\frac{\Phi_{0}-\Phi\left(\vec{r}_{r}\right)+2 G M / a_{\text {orb }}+2 V_{s}}{c^{2}}\right] \cdot \frac{1-\vec{n}_{r}^{s} \cdot \dot{\vec{r}}_{r} / c}{1-\vec{n}_{r}^{s} \cdot \dot{\vec{r}}^{s} / c} \\
& =f_{0} \cdot\left[1+\frac{\Phi_{0}-\Phi\left(\vec{r}_{r}\right)}{c^{2}}+\frac{2 G M}{c^{2}}\left(\frac{1}{a_{\text {orb }}}-\frac{1}{\left\|\vec{r}^{s}\right\|}\right)\right] \cdot \frac{1-\vec{n}_{r}^{s} \cdot \dot{\vec{r}}_{r} / c}{1-\vec{n}_{r}^{s} \cdot \dot{\vec{r}}^{s} / c}
\end{aligned}
$$

where $\omega$ is the angular velocity of the Earth's rotation, and the satellite gravitational potential is approximated by the point mass attraction.

In (9), the last term is due to special relativity, and the terms in the square brackets are the contributions to the Doppler frequency shift from general relativity. Inside the square brackets, the second term is the correction of the receiver potential difference from the geoid, and the third, i.e. $G M$ term is the effect from the orbit eccentricity, which will be elaborated later. Hence in a GPS observation system, extra frequency shifts are introduced due to the presence of the Earth's gravity field, the satellite orbital eccentricity and the receiver potential difference.

The special relativity term can be expanded into the following form

$$
\frac{1-\vec{n}_{r}^{s} \cdot \dot{\vec{r}}_{r} / c}{1-\vec{n}_{r}^{s} \cdot \dot{\vec{r}}^{s} / c}=\left(1-\vec{n}_{r}^{s} \cdot \dot{\vec{r}}_{r} / c\right) \cdot\left(1+\vec{n}_{r}^{s} \cdot \dot{\vec{r}}^{s} / c+\left(\dot{\vec{r}}^{s}\right)^{2} / c^{2}\right)=1+\frac{\vec{n}_{r}^{s} \cdot \dot{\vec{r}}_{r}^{s}}{c}+\frac{\dot{\vec{r}}^{s} \cdot \dot{\vec{r}}_{r}^{s}}{c^{2}}
$$

where $\dot{\vec{r}}_{r}^{s}=\dot{\vec{r}}^{s}-\dot{\vec{r}}_{r}$. Neglecting the last term in the right hand side of (10) and the terms in the brackets of (9), (9) agrees with the Doppler frequency shift under special relativity only.

Substituting (10) into (9), we have

$$
\begin{aligned}
f_{r}= & f_{0} \cdot\left[1+\frac{\Phi_{0}-\Phi\left(\vec{r}_{r}\right)}{c^{2}}+\frac{2 G M}{c^{2}}\left(\frac{1}{a_{o r b}}-\frac{1}{\left\|\vec{r}^{s}\right\|}\right)\right] \cdot\left(1+\frac{\vec{n}_{r}^{s} \cdot \dot{\vec{r}}_{r}^{s}}{c}+\frac{\dot{\vec{r}}^{s} \cdot \dot{\vec{r}}_{r}^{s}}{c^{2}}\right) \\
= & f_{0}+f_{0} \frac{\Phi_{0}-\Phi\left(\vec{r}_{r}\right)}{c^{2}}+f_{0} \frac{2 G M}{c^{2}}\left(\frac{1}{a_{o r b}}-\frac{1}{\left\|\vec{r}^{s}\right\|}\right)+f_{0} \cdot \frac{\vec{n}_{r}^{s} \cdot \dot{\vec{r}}_{r}^{s}}{c}+f_{0} \vec{n}_{r}^{s} \dot{\vec{r}}_{r}^{s} \frac{\left[\Phi_{0}-\Phi\left(\vec{r}_{r}\right)\right]}{c^{3}} \\
& +f_{0} \vec{n}_{r}^{s} \dot{\vec{r}}_{r}^{s} \frac{2 G M}{c^{3}}\left(\frac{1}{a_{o r b}}-\frac{1}{\left\|\vec{r}^{s}\right\|}\right)+f_{0} \frac{\dot{\vec{r}}^{s} \cdot \dot{\vec{r}}_{r}^{s}}{c^{2}}
\end{aligned}
$$


Rearranging (11) leads to

$$
\begin{aligned}
f_{r}-f_{0} & =f_{0} \cdot \frac{\vec{n}_{r}^{s} \cdot \dot{\vec{r}}_{r}^{s}}{c}+f_{0} \frac{\Phi_{0}-\Phi\left(\vec{r}_{r}\right)}{c^{2}}+f_{0} \frac{2 G M}{c^{2}}\left(\frac{1}{a_{o r b}}-\frac{1}{\left\|\vec{r}^{s}\right\|}\right)+f_{0} \frac{\dot{\vec{r}}^{s} \cdot \dot{\vec{r}}_{r}^{s}}{c^{2}} \\
& +f_{0} \vec{n}_{r}^{s} \dot{\vec{r}}_{r}^{s} \frac{\left[\Phi_{0}-\Phi\left(\vec{r}_{r}\right)\right]}{c^{3}}+f_{0} \vec{n}_{r}^{s} \dot{\vec{r}}_{r}^{s} \frac{2 G M}{c^{3}}\left(\frac{1}{a_{o r b}}-\frac{1}{\left\|\vec{r}^{s}\right\|}\right)
\end{aligned}
$$

Scaling the frequency shift into the velocity domain by multiplying the speed of light $c$, we have

$$
\begin{aligned}
D \lambda= & \vec{n}_{r}^{s} \cdot \dot{\vec{r}}_{r}^{s}+\frac{\Phi_{0}-\Phi\left(\vec{r}_{r}\right)}{c}+\frac{2 G M}{c}\left(\frac{1}{a_{o r b}}-\frac{1}{\left\|\vec{r}^{s}\right\|}\right)+\frac{\dot{\vec{r}}^{s} \cdot \dot{\vec{r}}_{r}^{s}}{c}+\vec{n}_{r}^{s} \dot{\vec{r}}_{r}^{s} \frac{\left[\Phi_{0}-\Phi\left(\vec{r}_{r}\right)\right]}{c^{2}} \\
& +\vec{n}_{r}^{s} \dot{\vec{r}}_{r}^{s} \frac{2 G M}{c^{2}}\left(\frac{1}{a_{o r b}}-\frac{1}{\left\|\vec{r}^{s}\right\|}\right) \\
= & {\left[\vec{n}_{r}^{s}+\frac{\dot{\vec{r}}^{s}}{c}+\vec{n}_{r}^{s} \frac{\left[\Phi_{0}-\Phi\left(\vec{r}_{r}\right)\right]}{c^{2}}+\vec{n}_{r}^{s} \frac{2 G M}{c^{2}}\left(\frac{1}{a_{o r b}}-\frac{1}{\left\|\vec{r}^{s}\right\|}\right)\right] \cdot \dot{\vec{r}}_{r}^{s}+\frac{\Phi_{0}-\Phi\left(\vec{r}_{r}\right)}{c}+\frac{2 G M}{c}\left(\frac{1}{a_{o r b}}-\frac{1}{\left\|\vec{r}^{s}\right\|}\right) } \\
= & {\left[\vec{n}_{r}^{s}+\frac{\dot{\vec{r}}^{s}}{c}\right] \cdot \dot{\vec{r}}_{r}^{s}+\frac{\Phi_{0}-\Phi\left(\vec{r}_{r}\right)}{c}+\frac{2 G M}{c}\left(\frac{1}{a_{o r b}}-\frac{1}{\left\|\vec{r}^{s}\right\|}\right) }
\end{aligned}
$$

where $\frac{\dot{\vec{r}}^{s}}{c}$ acts as a LOS direction correction applied to the range rate due to the satellite velocity. Equation (13) shows that the Doppler shift relates to a "direction changed" receiver to satellite range rate with relativistic biases of the receiver potential difference, and the satellite orbital eccentricity.

\section{Doppler Shift Observation Equation}

Thus far, we have established the inter-relationship of the theoretical Doppler shift and the relative motion between a satellite and a receiver, with the Earth gravity field considered. Because of the imperfection of the satellite clock and receiver clock, the presence of the non-vacuum media such as the ionosphere and troposphere, and other inherent errors in a GPS observation, there are other biases in the measured GPS Doppler shift. Taking account for all the effects mentioned above, the proposed Doppler shift observation equation is as follows

$$
\begin{aligned}
\lambda_{i} D_{r, i}^{s}(t) & =\left(\vec{n}_{r}^{s}+\frac{\dot{\vec{r}}^{s}}{c}\right) \cdot\left[\dot{\vec{r}}^{s}\left(t-\tau_{r}^{s}\right)-\dot{\vec{r}}_{r}(t)\right]-d \dot{I}_{r, i}^{s}+d \dot{T}_{r}^{s}+c \cdot d \dot{t}_{r}(t)-c \cdot d \dot{t}^{s}\left(t-\tau_{r}^{s}\right)+ \\
& \frac{\Phi_{0}-\Phi\left(\vec{r}_{r}\right)}{c}+\frac{2 G M}{c}\left(\frac{1}{a_{o r b}}-\frac{1}{\left\|\vec{r}^{s}\right\|}\right)+d \dot{R}_{\text {Sagnac }}+\varepsilon_{r}^{s}
\end{aligned}
$$

where subscript " $i$ " is used to designate frequency bands, " $l$ " for $\mathrm{L}_{1}$ and " 2 " for $\mathrm{L}_{2}$ respectively; $t$ is the measurement epoch, and $\tau_{r}^{s}$ is the signal propagation time from satellite " $s$ " to receiver " $r$ "; $d I, d T$ are the range delays in the ionosphere and 
troposphere; and $d t_{r}, d t^{s}$ are the receiver clock and satellite clock errors respectively; a dot over them is the corresponding change rate with respective to time, which is the error affects the Doppler shift measurement; $d R_{\text {Sagnac }}$ is the Sagnac correction for range measurement, and $d \dot{R}_{\text {Sagnac }}$ is the corresponding correction for the Doppler shift observable.

\section{Relativistic Corrections}

Note that an extra relativistic correction, i.e. the Sagnac Effect correction, is introduced in the above Doppler observation equation. The Sagnac Effect arises from the fact that a received signal has an extra signal passage during the signal propagation time due to the Earth rotation. There are other secondary relativistic effects in the GPS system that generate fractional frequency shifts, for example, tidal potentials from the sun and the moon, antenna phase wind-up, etc. Their effects on the instantaneous Doppler shift measurement are far less than $\pm 0.1 \mathrm{~mm} / \mathrm{s}$ and therefore can be considered neglected.

Thus we have three relativistic terms as biases in the observation model, and one relativistic term to change the direction of the line-of-sight unit vector.

\subsection{Receiver Potential Difference}

The term, $\frac{\Phi_{0}-\Phi\left(\vec{r}_{r}\right)}{c}$ is a site-dependent general relativistic correction term due to the receiver gravity potential. It becomes zero when a receiver is located rest on the geoid. Note that it is a common term in each Doppler shift measurement at a specific epoch and thus contributes to the user velocity in the same way as the receiver clock rate. Neglecting the receiver potential difference will cause a biased receiver clock rate estimate, but do little harm to the user velocity estimation. This may provide the reason that this term has been historically neglected for velocity determination using GPS.

The potential difference can be simply estimated by the approximation of $\Phi_{0}-\Phi\left(\vec{r}_{r}\right)=g H_{r}$ where $g$ is the gravity acceleration and $H_{r}$ is the height of a receiver. The maximum effect of this term on the earth surface is at the peak of Mt. Everest, which is less than $0.3 \mathrm{~mm} / \mathrm{s}$. The magnitude of this term varies with receiver heights; it increases when in aviation and space applications.

\subsection{Orbital Eccentricity}

This relativistic effect correction for the range of receiver $r$ and satellite $s$ depends on the satellite orbit. It can be expressed in terms of the satellite position and velocity as (ARINC 2000, p.89) 
$d R_{\text {eccentricity }}=-\frac{2}{c} \cdot \vec{r}^{s} \bullet \dot{\vec{r}}^{s}$

This correction is not sensitive to whether the vectors of position and velocity are expressed in earth-fixed rotating coordinate system or in earth-centred inertial coordinate system (ibid). The eccentricity correction for range measurement is a periodic function and is the biggest correction in GPS relativity. Simply differentiating the above equation with respect to time gives

$d \dot{R}_{\text {eccentricity }}=-\frac{2}{c} \cdot\left[\left(\dot{\vec{r}}^{s}\right)^{2}+\vec{r}^{s} \cdot \ddot{\vec{r}}^{s}\right]$

where $\ddot{\ddot{r}}^{s}$ is the satellite acceleration. Since a satellite conserves energy while in orbit, according to Vis-Viva equation (Montenbruck and Gill 2000, p.20), we have

$\left(\dot{\vec{r}}^{s}\right)^{2}=G M\left(\frac{2}{\|\vec{r}\|}-\frac{1}{a_{\text {orb }}}\right)$

and the satellite acceleration is, for example (Misra and Enge 2001, p.99)

$\ddot{\vec{r}}^{s}=-\frac{G M}{\left\|\vec{r}^{s}\right\|^{3}} \vec{r}^{s}$

Substituting (17) and (18) into the brackets of (16) leads to

$d \dot{R}_{\text {eccentricity }}=-\frac{2}{c} \cdot\left[\left(\dot{\vec{r}}^{s}\right)^{2}+\vec{r}^{s} \cdot \ddot{\vec{r}}^{s}\right]=\frac{2 G M}{c}\left(\frac{1}{a_{\text {orb }}}-\frac{1}{\left\|\vec{r}^{s}\right\|}\right)$

This proves that the term $\frac{2 G M}{c}\left(\frac{1}{a_{o r b}}-\frac{1}{\left\|\vec{r}^{s}\right\|}\right)$ is the relativistic correction for the orbit eccentricity. The eccentricity correction is more intuitive in the frequency (velocity) domain than in the range or time domain (see Eq.(15)), since this term vanishes in a circular orbit.

\subsection{Earth Rotation Correction (Sagnac Effect)}

As mentioned previously, the rotation of the Earth during the GPS signal propagation period causes a relativistic error that is known as the Sagnac effect (Ashby and Spilker 1996; Leva et al. 1996). The incoming signal has an extra signal passage.

The Sagnac Effect correction given by Ashby and Spilker (1996) is in the form of

$d R_{\text {Sagnac }}=\frac{2 \vec{\Omega}_{e} \cdot \vec{A}}{c}$

where $\vec{\Omega}_{e}=(0,0, \omega)^{\mathrm{T}}$ is the vector of the angular rate of the Earth rotation and $\vec{A}$ is the shaded area of the triangles swept out by an arrow with its tail at the Earth centre and its head following the electromagnetic signals. The Sagnac correction for range measurement can be calculated using 
$d R_{\text {Sagnac }}=\frac{2 \Omega_{e}{ }^{T}}{c} \cdot \frac{\vec{r}_{r} \times \vec{r}^{s}}{2}=\frac{\omega}{c}\left(y^{s} x_{r}-x^{s} y_{r}\right)$

which is identical to the aberration correction formula given by Seeber (2003, p.198). The Sagnac correction for Doppler may then be obtained by differentiating the above equation with respect to time.

$$
d \dot{R}_{\text {Sagnac }}=\frac{\Omega_{e}{ }^{T}}{c} \cdot \frac{d}{d t}\left(\vec{r}_{r} \times \vec{r}^{s}\right)=\frac{\omega}{c}\left[\dot{x}^{s} y^{s}-\dot{y}_{r} x^{s}+x_{r} \cdot \dot{y}^{s}-y^{s} \dot{x}^{s}\right]
$$

\subsection{Line-of-Sight Correction}

This is the correction applied to a receiver-satellite line-of-sight unit vector to account for the relativistic effect introduced by the high speed of the satellite in orbit (special relativity). As can be seen from (14), the "received" receiver-satellite range rate differs from the line-of-sight direction by $\frac{\dot{\vec{r}}^{s}}{c}$. This correction changes with the satellite velocity over time. Since it changes the line-of-sight direction, we name it as the line-of-sight correction. The contributions of the line-of-sight correction to the user velocity estimation are mainly in the vertical direction, because under good satellite geometry the horizontal errors will be almost averaged out. However, bias of velocity estimation in horizontal directions may be introduced when the satellite geometry is poor.

\section{Concluding Remarks}

The proposed model highlights two relativistic terms for precise velocity determinations using GPS. The relativistic Doppler error due to the receiver potential difference applies to all the Doppler shifts measured at the same epoch, thus resulting in a biased receiver clock rate estimate. The high-speed motion of a satellite affects the LOS unit vector thus changing the direction of the range rate. As a result, a user velocity will be biased, mainly in the zenith direction.

This paper also presents a highly accurate function model to relate a GPS Doppler frequency shift measurement to a receiver-to-satellite relative velocity. The new Doppler shift observation equation allows a user velocity to be accurately determined anywhere on or above the Earth, in either static or high dynamic applications.

Theoretically the relativistic terms in Doppler observation should go into the carrier phase measurements in some form since the carrier phase is the integrated Doppler over time. This suggests that there might be corresponding terms in the one-way carrier phase measurement as well. The authors believe that this would be a topic worthy of further investigation. Of particular interests are the effects from the lineof-sight corrections, i.e. does the LOS direction change in the range observation? 
Such investigations may be of benefit to the accuracy improvement of precise point positioning.

\section{Appendix}

Since all the contributions of this paper are based on (9), a variation form of (7) given by Ashby (2003), for completeness, a proof is provided as follows to justify the derived formulae and the conclusions thereafter.

The range from satellite $s$ to receiver $r$ is

$\rho=\vec{r}^{s}-\vec{r}_{r}$

From (A1), we have the difference between the coordinate time of reception and the coordinate time of transmission as

$t_{r}-t^{s}=\frac{\rho}{c}$

Differentiating (A2) with respect to time, we have

$1-\frac{d t^{s}}{d t_{r}}=\frac{1}{c}\left(\frac{\partial \rho}{\partial t_{r}}+\frac{\partial \rho}{\partial t^{s}} \frac{d t^{s}}{d t_{r}}\right)=\frac{1}{c}\left(\vec{n}_{r}^{s} \cdot \dot{\vec{r}}_{r}-\vec{n}_{r}^{s} \cdot \dot{\vec{r}}^{s} \frac{d t^{s}}{d t_{r}}\right)$

This can be formulated as

$\frac{d t^{s}}{d t_{r}}=\frac{1-\vec{n}_{r}^{s} \cdot \dot{\vec{r}}_{r} / c}{1-\vec{n}_{r}^{s} \cdot \dot{\vec{r}}^{s} / c}$

The ratio of the received frequency $f_{r}$ and the transmitted frequency $f_{s}$ can be expressed

$$
\frac{f_{r}}{f_{s}}=\frac{d \tau^{s}}{d \tau_{r}}=\frac{\frac{d t_{r}}{d \tau_{r}}}{\frac{d t^{s}}{d \tau^{s}}} \frac{d t^{s}}{d t_{r}}
$$

At every space-time point in weak field, the proper time $\tau$ relates to the coordinate time $t$ by, see (Ashby and Spilker 1996, p.655)

$d \tau=\sqrt{-g_{00}(\vec{r})} \cdot d t$

where $g_{00}$ is the classical approximation of the zero-zero component of the metric

tensor. In a rotating frame such as the ECEF system (Ashby, 2003, p.12)

$g_{00}(\vec{r})=-\left[1+\frac{2 \Phi(\vec{r})}{c^{2}}\right]=-\left[1+\frac{2 V}{c^{2}}-\frac{\omega^{2}\|\vec{r}\|^{2} \cos B}{c^{2}}\right]$

where $\Phi$ is the classical effective Newtonian gravitational potential including the centrifugal potential, Note that the kinetic energy term is neglected. Substituting $g_{00}$ into (A6) and expanding it with binomials lead to 


$$
\frac{d t}{d \tau}=\left[1+\frac{2 \Phi(\vec{r})}{c^{2}}\right]^{-\frac{1}{2}}=1-\frac{\Phi(\vec{r})}{c^{2}}=1-\frac{V(\vec{r})-\frac{1}{2} \omega^{2}\|\vec{r}\|^{2} \cos ^{2} B}{c^{2}}
$$

For satellite $s$, we keep the ratio in the following form

$$
\frac{d t^{s}}{d \tau_{s}}=1-\frac{V\left(\vec{r}^{s}\right)-\frac{1}{2}\left\|\dot{\vec{r}}^{s}\right\|^{2}}{c^{2}}
$$

With equations (A8), (A9), and (A4), the relationship of the received frequency $f_{r}$ and the transmitted frequency $f_{s}$ can then be written as

$$
\begin{aligned}
f_{r} & =f_{s} \cdot\left[\frac{1-1 / c^{2}\left[V_{r}-1 / 2 \cdot \omega^{2}\left\|\vec{r}_{r}\right\|^{2}(\cos B)^{2}\right]}{1-1 / c^{2}\left[V\left(\vec{r}^{s}\right)-\frac{1}{2}\left\|\dot{\vec{r}}^{s}\right\|^{2}\right]}\right] \cdot \frac{1-\vec{n}_{r}^{s} \cdot \dot{\vec{r}}_{r} / c}{1-\vec{n}_{r}^{s} \cdot \dot{\vec{r}}^{s} / c} \\
& =f_{s}\left[\left(1-\frac{\Phi\left(\vec{r}_{r}\right)}{c^{2}}\right)\left(1+\frac{V^{s}-1 / 2\left\|\dot{\vec{r}}^{s}\right\|^{2}}{c^{2}}\right)\right] \frac{1-\vec{n}_{r}^{s} \cdot \dot{\vec{r}}_{r} / c}{1-\vec{n}_{r}^{s} \cdot \dot{\vec{r}}^{s} / c}
\end{aligned}
$$

The above equation can be simplified as

$$
f_{r}=f_{s}\left[1+\frac{-\Phi\left(\vec{r}_{r}\right)}{c^{2}}+\frac{V^{s}-1 / 2\left\|\dot{\vec{r}}^{s}\right\|^{2}}{c^{2}}\right] \cdot \frac{1-\vec{n}_{r}^{s} \cdot \dot{\vec{r}}_{r} / c}{1-\vec{n}_{r}^{s} \cdot \dot{\vec{r}}^{s} / c}
$$

Since the sum of the potential energy and the kinetic energy for a satellite is constant during motion in it orbit (Montenbruck and Gill 2000, p.20), this gives

$$
V^{s}+1 / 2\left\|\dot{\vec{r}}^{s}\right\|^{2}=-\frac{G M}{2 a_{\text {orb }}} \Rightarrow 1 / 2\left\|\dot{\vec{r}}^{s}\right\|^{2}=-\frac{G M}{2 a_{\text {orb }}}-V^{s}
$$

Substituting (A12) into (A11), we have

$$
f_{r}=f_{s}\left[1+\frac{-\Phi\left(\vec{r}_{r}\right)}{c^{2}}+\frac{2 V^{s}+G M / 2 a_{o r b}}{c^{2}}\right] \cdot \frac{1-\vec{n}_{r}^{s} \cdot \dot{\vec{r}}_{r} / c}{1-\vec{n}_{r}^{s} \cdot \dot{\vec{r}}^{s} / c}
$$

The GPS system has already adopted a frequency adjustment (Ashby and Spilker 1996, p.679) by

$$
f_{s}=f_{0}\left[1+\frac{3 G M}{2 a_{\text {orb }} c^{2}}+\frac{\Phi_{0}}{c^{2}}\right]
$$

where $f_{0}$ is the nominal GPS frequency. After incorporating this frequency adjustment, equation (A13) changes to 


$$
\begin{aligned}
f_{r} & =f_{0}\left[1+\frac{\Phi_{0}-\Phi\left(\vec{r}_{r}\right)}{c^{2}}+\frac{2 G M / a_{o r b}+2 V^{s}}{c^{2}}\right] \cdot \frac{1-\vec{n}_{r}^{s} \cdot \dot{\vec{r}}_{r} / c}{1-\vec{n}_{r}^{s} \cdot \dot{\vec{r}}^{s} / c} \\
& =f_{0}\left[1+\frac{\Phi_{0}-\Phi\left(\vec{r}_{r}\right)}{c^{2}}+\frac{2 G M}{c^{2}}\left(\frac{1}{a_{o r b}}-\frac{1}{\left\|\vec{r}^{s}\right\|}\right)\right] \cdot \frac{1-\vec{n}_{r}^{s} \cdot \dot{\vec{r}}_{r} / c}{1-\vec{n}_{r}^{s} \cdot \dot{\vec{r}}^{s} / c}
\end{aligned}
$$

which is exactly the form of (9).

\section{Acknowledgement}

This research presented is partially supported by the Cooperative Research Centre (CRC) for microTechnology, Australia. Consultation with Prof. Gunter Seeber, and constructive comments from Prof. Juergen Mueller from the University of Hannover, Dr. Drazen Svehla from the Technical University of Munich and Prof Will Featherstone from Curtin University of Technology are acknowledged and greatly appreciated.

\section{References}

ARINC (2000). Interface Control Document, Navstar GPS Space Segment/Navigation User Interfaces, ICDGPS-200, Revision C. Arinc Research Corporation

Ashby N (2003). Relativity in the Global Positioning System. Living Rev. Relativity 6, Online article, http://www.livingreviews.org/Articles/Volum6/2003-lashby. Accessed on 08/12/2004

Ashby N, Spilker JJ (1996). Introduction to Relativistic Effects on the Global Positioning System. In Parkinson B. W. (ed). Global Positioning System: Theory and Applications. AIAA, Inc. Vol(1): pp: 623-698

Audoin C, Guinot B (2001). The Measurement of Time: Time, Frequency and the Atomic Clock. Cambridge University Press, Cambridge, New York

DoD (1996). NAVSTAR GPS User Equipment Introduction. Rep. public release version, Department of Defense, USA

Hofmann-Wellenhof B, Lichtenegger H, and Collins J (2001). Global Positioning System Theory and Practice, Springer-Verlag, Wien New York

IAU Res (2000). IAU Resolutions Adopted at the 24th General Assembly. http://danof.obspm.fr/IAU_resolutions/Resol-UAI.htm. Accessed on 08/12/2004

Kouba J (2002). Relativistic Time Transformations in GPS. GPS Solutions 5(4): pp:1-9

Kouba J (2004). Improved Relativistic Transformations in GPS. GPS Solutions 8(3): pp:170-180

Leva J, Haag M and Dyke K (1996). Performance of Standalone GPS. In Kaplan E. D. (ed) Understanding GPS: Principles and Applications. Artech House, Boston London, pp: 237-320

Misra P, Enge P (2001). Global Positioning System: Signals, Measurements, and Performance. Ganga-Jamuna Press, Lincoln, Massachusetts

Montenbruck O, Gill E (2000). Satellite Orbits: Models, Methods, and Applications. Springer-Verlag, Heidelberg

NIMA (2000). World Geodetic System 1984: Its Definition and Relationships with Local Geodetic Systems. Rep. NIMA TR8350.2, National Imagery and Mapping Agency

Seeber G (1993). Satellite Geodesy: Foundations, Methods, and Applications Walter de Gruyter, Berlin

Seeber G (2003). Satellite Geodesy. Walter de Gruyter GmbH \& Co, Berlin

Serrano L, Kim D, Langley R. B, Itani K and Ueno M (2004). A GPS Velocity Sensor: How Accurate Can It Be? A First Look. Proceedings of ION-NTM 2004, San Diego, California, USA, pp:875-885

Serway R (1996). Physics for Scientists \& Engineers with Modern Physics. Saunders College Publishing, Tokyo

van Graas F and Soloview A (2003). Precise Velocity Estimation Using a Stand-Alone GPS Receiver. Proceedings of ION-NTM 2003m, pp.262-271, Anaheim, California, USA

Wells D., Beck B, Delikaraoglou D, Kleusberg A, Krakiwsky E, Lachapelle G, Langley R, Nakiboglu M, Schwarz K, Tranquilla J and Vanicek P (1987). Guide to GPS Positioning. Canadian GPS Associates, Fredericton, New Brunswick, Canada 